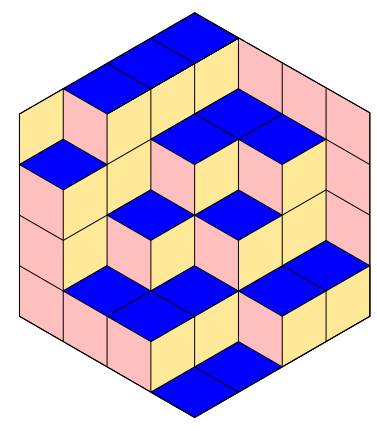

Nicholas Proudfoot

Equivariant Kazhdan-Lusztig polynomials of $q$-niform matroids

Volume 2, issue 4 (2019), p. 613-619.

<http://alco.centre-mersenne.org/item/ALCO_2019__2_4_613_0>

(C) The journal and the authors, 2019.

Some rights reserved.

(c) BY This article is licensed under the

Creative Commons ATtribution 4.0 InTERnational License.

http://creativecommons.org/licenses/by/4.0/

Access to articles published by the journal Algebraic Combinatorics on the website http://alco.centre-mersenne.org/ implies agreement with the Terms of Use (http://alco.centre-mersenne.org/legal/).

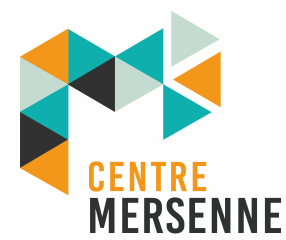

Algebraic Combinatorics is member of the Centre Mersenne for Open Scientific Publishing www.centre-mersenne.org 


\title{
Equivariant Kazhdan-Lusztig polynomials of $q$-niform matroids
}

\author{
Nicholas Proudfoot
}

\begin{abstract}
We study $q$-analogues of uniform matroids, which we call $q$-niform matroids. While uniform matroids admit actions of symmetric groups, $q$-niform matroids admit actions of finite general linear groups. We show that the equivariant Kazhdan-Lusztig polynomial of a $q$-niform matroid is the unipotent $q$-analogue of the equivariant Kazhdan-Lusztig polynomial of the corresponding uniform matroid, thus providing evidence for the positivity conjecture for equivariant Kazhdan-Lusztig polynomials.
\end{abstract}

\section{INTRODUCTION}

For any matroid $M$, the Kazhdan-Lusztig polynomial $P_{M}(t) \in \mathbb{Z}[t]$ was introduced in [7]. In the case where the matroid $M$ admits the action of a finite group $W$, one can define the equivariant Kazhdan-Lusztig polynomial $P_{M}^{W}(t)$ [10]; this is a polynomial whose coefficients are virtual representations of $W$ (in characteristic zero) with dimensions equal to the coefficients of $P_{M}(t)$.

Though these polynomials admit elementary recursive definitions, there are not many families of matroids for which explicit formulas are known. Non-equivariant formulas exist for thagomizer matroids [11] and fan, wheel, and whirl matroids [15]. Kazhdan-Lusztig polynomials of braid matroids have been studied extensively, both in the equivariant [18] and non-equivariant [14] settings, though no simple formulas have been obtained.

The most interesting explicit formulas that we have are for uniform matroids. Let $U_{n, m}$ be the uniform matroid of rank $n-m$ on a set of cardinality $n$, which admits an action of the symmetric group $S_{n}$. For any partition $\lambda$ of $n$, let $V[\lambda]$ be the associated irreducible representation of $S_{n}$. The following theorem was proved in [10, Theorem 3.1]; an independent proof of the non-equivariant statement was later given in $[9$, Theorem 1.2].

TheOrem 1.1. Let $C_{n, m}^{i}$ be the coefficient of $t^{i}$ in the $S_{n}$-equivariant Kazhdan-Lusztig polynomial of $U_{n, m}$, and let $c_{n, m}^{i}:=\operatorname{dim} C_{n, m}^{i}$ be the corresponding non-equivariant coefficient.

Manuscript received 1st September 2018, revised 18th January 2019, accepted 14th January 2019. KEYWORDS. Kazhdan-Lusztig polynomial, matroid, unipotent representation.

ACKNOWLEDGEMENTS. The author is supported by NSF grant DMS-1565036. 
- $C_{n, m}^{0}=V[n]$, and for all $i>0$,

$$
C_{n, m}^{i}=\sum_{b=1}^{\min (m, n-m-2 i)} V\left[n-2 i-b+1, b+1,2^{i-1}\right] .
$$

- $c_{n, m}^{0}=1$, and for all $i>0, c_{n, m}^{i}$ is equal to

$$
\sum_{b=1}^{\min (m, n-m-2 i)} \frac{(n-2 i-2 b+1) n !}{(n-i-b)(n-i-b+1)(i+b)(i+b-1)(n-2 i-b) !(b-1) ! i !(i-1) !} .
$$

The purpose of this note is to obtain a $q$-analogue of Theorem 1.1. Let $q$ be a prime power, and let $U_{n, 0}(q)$ be the rank $n$ matroid associated with the collection of all hyperplanes in the vector space $\mathbb{F}_{q}^{n}$, which we regard as a $q$-analogue of the Boolean matroid of rank $n$. For any natural number $m \leqslant n$, let $U_{n, m}(q)$ be the truncation of $U_{n, 0}(q)$ to rank $n-m$. More concretely, a basis for $U_{n, m}(q)$ is a set of $n-m$ hyperplanes whose intersection has dimension $m$. The matroid $U_{n, m}(q)$ is a $q$-analogue of the uniform matroid $U_{n, m}$, and we will therefore refer to it as a $q$ niform matroid. This matroid was also studied in [12], where the authors computed the Hilbert series of its Chow ring. The $q$-niform matroid $U_{n, m}(q)$ admits a natural action of the group $\mathrm{GL}_{n}(q)$ of invertible $n \times n$ matrices with coefficients in $\mathbb{F}_{q}$, which is a $q$-analogue of $S_{n}$.

The representation theory of $\mathrm{GL}_{n}(q)$ is much more complicated than the representation theory of $S_{n}$. However, there is a certain subset of irreducible representations of $\mathrm{GL}_{n}(q)$, known as irreducible unipotent representations, that correspond bijectively to the irreducible representations of $S_{n}$. For any partition $\lambda$ of $n$, let $V(q)[\lambda]$ be the associated irreducible unipotent representation of $\operatorname{GL}_{n}(q)$, which we will refer to as the unipotent q-analogue of $V[\lambda]$. For any positive integer $k$, we use the standard notation

$$
[k]_{q}:=1+q+\cdots+q^{k-1} \quad \text { and } \quad[k]_{q} !:=[k]_{q}[k-1]_{q} \cdots[1]_{q} .
$$

The following theorem, which is our main result, says that the equivariant KazhdanLusztig coefficients of $U_{n, m}(q)$ are precisely the unipotent $q$-analogues of the equivariant Kazhdan-Lusztig coefficients of $U_{n, m}$.

THEOREM 1.2. Let $C_{n, m}^{i}(q)$ be the coefficient of $t^{i}$ in the $\mathrm{GL}_{n}(q)$-equivariant KazhdanLusztig polynomial of $U_{n, m}(q)$, and let $c_{n, m}^{i}(q):=\operatorname{dim} C_{n, m}^{i}(q)$ be the corresponding non-equivariant coefficient.

- $C_{n, m}^{0}(q)=V(q)[n]$, and for all $i>0$,

$$
C_{n, m}^{i}(q)=\sum_{b=1}^{\min (m, n-m-2 i)} V(q)\left[n-2 i-b+1, b+1,2^{i-1}\right] .
$$

- $c_{n, m}^{0}(q)=1$, and for all $i>0, c_{n, m}^{i}(q)$ is equal to

$$
\sum_{b=1}^{\min (m, n-m-2 i)} \frac{q^{b-1+i(i+1)}[n-2 i-2 b+1]_{q}[n]_{q} !}{[n-i-b]_{q}[n-i-b+1]_{q}[i+b]_{q}[i+b-1]_{q}[n-2 i-b]_{q} ![b-1]_{q} ![i]_{q} ![i-1]_{q} !} .
$$

REMARK 1.3. For any matroid $M$, the coefficients of $P_{M}(t)$ are conjectured to be non-negative [7, Conjecture 2.3]. More generally, the coefficients of $P_{M}^{W}(t)$ are conjectured to be honest (rather than virtual) representations of $W$ [10, Conjecture 2.13]. These conjectures are proved when $M$ is realizable [7, Theorem 3.10] (respectively equivariantly realizable [10, Corollary 2.12]), but no proof exists in the general case. The matroid $U_{n, m}$ is always realizable, but it is not equivariantly realizable unless 
$m \in\{0,1, n-1, n\}$ (of these, only the $m=1$ case yields nontrivial Kazhdan-Lusztig coefficients). Similarly, the matroid $U_{n, m}(q)$ is always realizable, but it is typically not equivariantly realizable. Thus Theorems 1.1 and 1.2 both provide significant evidence for the equivariant non-negativity conjecture.

REMARK 1.4. Theorem 1.1 implies that $\left\{C_{n, m}^{i} \mid n \geqslant m\right\}$ admits the structure of a finitely generated FI-module [3, Theorem 1.13], while Theorem 1.2 implies that $\left\{C_{n, m}^{i}(q) \mid n \geqslant m\right\}$ admits the structure of a finitely generated VI-module [8, Theorem 1.6]. In order to define these structures in a natural way, we would need to be able to define $C_{n, m}^{i}$ and $C_{n, m}^{i}(q)$ as actual vector spaces rather than as isomorphism classes of vector spaces. The matroid $U_{n, 1}$ is equivariantly realizable, which means that we have a cohomological interpretation of $C_{n, 1}^{i}$, and we obtain a canonical $\mathrm{FI}^{\mathrm{op}}$-module structure from [18, Theorem 3.3(1)]; dualizing then gives a canonical finitely generated FI-module. In joint work with Braden, Huh, Matherne, and Wang, the author is working to construct a canonical vector space isomorphic to the coefficient of $t^{i}$ in $P_{M}(t)$ for any matroid $M$. When this goal is achieved, we believe that this construction will induce a canonical $\mathrm{FI}^{\mathrm{op}}$-module structure on $\left\{C_{n, m}^{i} \mid n \geqslant m\right\}$ and a canonical $\mathrm{VI}^{\mathrm{op}}$-module structure on $\left\{C_{n, m}^{i}(q) \mid n \geqslant m\right\}$, each with finitely generated duals.

Our proof of Theorem 1.2 relies heavily on Theorem 1.1 along with the Comparison Theorem (Theorem 2.1), which roughly says that calculations involving HarishChandra induction of unipotent representations of finite general linear groups are essentially equivalent to the analogous calculations for symmetric groups. The only additional ingredients in the proof are to check that the Orlik-Solomon algebra of $U_{n, m}(q)$ is the unipotent $q$-analogue of the Orlik-Solomon algebra of $U_{n, m}$ (Example 3.4) and that the recursive formula for $C_{n, m}^{i}(q)$ is essentially the same as the recursive formula for $C_{n, m}^{i}$ (Equations (7) and (8)).

\section{Unipotent Representations And the Comparison Theorem}

Given a pair of natural numbers $k \leqslant n$ and a pair of representations $V$ of $S_{k}$ and $V^{\prime}$ of $S_{n-k}$, we define

$$
V * V^{\prime}:=\operatorname{Ind}_{S_{k} \times S_{n-k}}^{S_{n}}\left(V \otimes V^{\prime}\right) .
$$

Irreducible representations of the symmetric group $S_{n}$ are classified by partitions of $n$. Given a partition $\lambda$, let $V[\lambda]$ be the associated representation. For each cell $(i, j)$ in the Young diagram for $\lambda$, let $h_{\lambda}(i, j)$ be the corresponding hook length; then the dimension of $V[\lambda]$ is equal to

$$
\frac{n !}{\prod h_{\lambda}(i, j)} .
$$

We now review some analogous statements and constructions in the representation theory of finite general linear groups. Given a pair of natural numbers $k \leqslant n$, let $P_{k, n}(q) \subset \mathrm{GL}_{n}(q)$ denote the parabolic subgroup associated with the Levi $\mathrm{GL}_{k}(q) \times$ $\mathrm{GL}_{n-k}(q)$. Given a pair of representations $V(q)$ of $\mathrm{GL}_{k}(q)$ and $V^{\prime}(q)$ of $\mathrm{GL}_{n-k}(q)$, we obtain a representation $V(q) \otimes V^{\prime}(q)$ of $\mathrm{GL}_{k}(q) \times \mathrm{GL}_{n-k}(q)$, and we may interpret this as a representation of $P_{k, n}(q)$ via the natural surjection $P_{k, n}(q) \rightarrow \mathrm{GL}_{k}(q) \times \mathrm{GL}_{n-k}(q)$. We then define

$$
V(q) * V^{\prime}(q):=\operatorname{Ind}_{P_{k, n}(q)}^{\mathrm{GL}_{n}(q)}\left(V(q) \otimes V^{\prime}(q)\right) .
$$

This operation is called Harish-Chandra induction.

Let $\mathrm{B}_{n}(q) \subset \mathrm{GL}_{n}(q)$ be the subgroup of upper triangular matrices. An irreducible representation of $\mathrm{GL}_{n}(q)$ is called unipotent if it appears as a direct summand of the 
representation

$$
\mathbb{C}\left[\mathrm{GL}_{n}(q) / \mathrm{B}_{n}(q)\right]=\operatorname{Ind}_{\mathrm{B}_{n}(q)}^{\mathrm{GL}_{n}(q)}\left(\operatorname{tric}_{\mathrm{GL}_{n}(q)}\right) .
$$

(We note that the definition of unipotent representations of finite groups of Lie type outside of type A is more complicated.) An arbitrary representation is called unipotent if it is isomorphic to a direct sum of irreducible unipotent representations.

THEOREM 2.1. Let $q$ be a prime power and $n$ a natural number.

(1) Irreducible unipotent representations of $\mathrm{GL}_{n}(q)$ are in canonical bijection with partitions of $n$.

(2) The irreducible unipotent representation $V(q)[\lambda]$ associated with the partition $\lambda$ has dimension

$$
q^{\sum(k-1) \lambda_{k}} \frac{[n]_{q} !}{\prod\left[h_{\lambda}(i, j)\right]_{q}} .
$$

(3) If $k \leqslant n, V(q)$ is a unipotent representation of $\mathrm{GL}_{k}(q)$, and $V^{\prime}(q)$ is a unipotent representation of $\mathrm{GL}_{n-k}(q)$, then $V(q) * V^{\prime}(q)$ is a unipotent representation of $\mathrm{GL}_{n}(q)$.

(4) Let $\lambda, \mu$, and $\nu$ be partitions of $n, k$, and $n-k$, respectively. The multiplicity of $V(q)[\lambda]$ in $V(q)[\mu] * V(q)[\nu]$ is equal to the multiplicity of $V[\lambda]$ in $V[\mu] * V[\nu]$.

Proof. Statements 1 and 4 appear in [4, Theorem B]. The fact that the dimension of $V(q)[\lambda]$ is polynomial in $q$ appears in [2, Theorem 2.6]. For an explicit calculation of this polynomial, see [5, Equation (1.1)]. Finally, Statement 3 follows from the fact that $\mathbb{C}\left[\mathrm{GL}_{k}(q) / \mathrm{B}_{k}(q)\right] * \mathbb{C}\left[\mathrm{GL}_{n-k}(q) / \mathrm{B}_{n-k}(q)\right] \cong \mathbb{C}\left[\mathrm{GL}_{n}(q) / \mathrm{B}_{n}(q)\right]$.

REMARK 2.2. The standard proof of Theorem 2.1(1) is very far from constructive. One proves that the endomorphism algebra of $\mathbb{C}\left[\mathrm{GL}_{n}(q) / \mathrm{B}_{n}(q)\right]$ is isomorphic to the Hecke algebra of $S_{n}$; this implies that the irreducible constituents of $\mathbb{C}\left[\mathrm{GL}_{n}(q) / \mathrm{B}_{n}(q)\right]$ are in canonical bijection with irreducible modules over the Hecke algebra, which are in turn in canonical bijection with irreducible representations of $S_{n}$. However, a recent paper of Andrews [1] gives a construction of $V(q)[\lambda]$ modeled on tableaux, which is analogous to the usual construction of $V[\lambda]$.

REMARK 2.3. A generalization of Statement 4 due to Howlett and Lehrer [13, Theorem 5.9] is commonly referred to as the Comparison Theorem. For the purposes of this paper, we will use this terminology to refer to the entirety of Theorem 2.1.

\section{ORLIK-SOLOMON ALGEBRAS}

For any matroid $M$ on the ground set $E$, let $O S_{M}^{*}$ be the Orlik-Solomon algebra of $M[17]$, and let

$$
\chi_{M}(t):=\sum_{i=0}^{\mathrm{rk} M}(-1)^{i} \operatorname{dim} O S_{M}^{i} t^{\mathrm{rk} M-i}
$$

be the characteristic polynomial of $M$. The Orlik-Solomon algebra is a quotient of the exterior algebra over the complex numbers with generators $\left\{x_{e} \mid e \in E\right\}$. Let $\overline{O S}_{M}^{*}$ be the reduced Orlik-Solomon algebra of $M$, which is defined as the subalgebra of $O S_{M}^{*}$ generated by $\left\{x_{e}-x_{e^{\prime}} \mid e, e^{\prime} \in E\right\}$. If $\operatorname{rk} M>0$, then we have a graded algebra isomorphism

$$
O S_{M}^{*} \cong \overline{O S}_{M}^{*} \otimes \mathbb{C}[x] /\left\langle x^{2}\right\rangle
$$

and therefore a vector space isomorphism

$$
O S_{M}^{i} \cong \overline{O S}_{M}^{i} \oplus \overline{O S}_{M}^{i-1}
$$


If a finite group $W$ acts on $M$, we obtain induced actions on $O S_{M}^{*}$ and $\overline{O S}_{M}^{*}$, and the isomorphisms of Equations (1) and (2) are $W$-equivariant.

EXAMPLE 3.1. Suppose that $V$ is a vector space over $\mathbb{F}_{q}$, and that $\left\{H_{e} \mid e \in E\right\}$ is a collection of hyperplanes with associated matroid $M$. Fix a prime $\ell$ that does not divide $q$, and fix an embedding of $\mathbb{Q}_{\ell}$ into $\mathbb{C}$. Let

$$
X:=V\left(\overline{\mathbb{F}}_{q}\right) \backslash \bigcup_{e \in E} H_{e}\left(\overline{\mathbb{F}}_{q}\right) \quad \text { and } \quad \mathbb{P} X:=\mathbb{P} V\left(\overline{\mathbb{F}}_{q}\right) \backslash \bigcup_{e \in E} \mathbb{P} H_{e}\left(\overline{\mathbb{F}}_{q}\right) .
$$

Then we have canonical isomorphisms

$$
O S_{M}^{*} \cong H^{*}\left(X ; \mathbb{Q}_{\ell}\right) \otimes_{\mathbb{Q}_{\ell}} \mathbb{C} \quad \text { and } \quad \overline{O S}_{M}^{*} \cong H^{*}\left(\mathbb{P} X ; \mathbb{Q}_{\ell}\right) \otimes_{\mathbb{Q}_{\ell}} \mathbb{C},
$$

where the cohomology rings are $\ell$-adic étale cohomology. If $\operatorname{rk} M>0$, then we have an isomorphism $X \cong \mathbb{P} X \times \mathbb{G}_{m}\left(\overline{\mathbb{F}}_{q}\right)$, and Equation (1) is simply the Kunneth formula. If $W$ acts on $V$ by linear automorphisms preserving the collection of hyperplanes, we obtain an induced action on $M$, and these isomorphisms are $W$-equivariant.

EXAMPLE 3.2. The Boolean matroid $U_{n, 0}$ is $S_{n}$-equivariantly realized by the coordinate hyperplanes in $\mathbb{F}_{q}^{n}$. Its Orlik-Solomon algebra $O S_{n, 0}^{*}$ is equal to the exterior algebra on $n$ generators, which is isomorphic to the cohomology of $X_{n, 0} \cong \mathbb{G}_{m}^{n}\left(\mathbb{F}_{q}\right)$. As a representation of $S_{n}$, we have

$$
O S_{n, 0}^{*} \cong \Lambda^{*}(V[n-1,1] \oplus V[n]) \quad \text { and } \quad \overline{O S}_{n, 0}^{*} \cong \Lambda^{*}(V[n-1,1]) .
$$

In particular, this implies that

$$
\overline{O S}_{n, 0}^{i} \cong V\left[n-i, 1^{i}\right]
$$

for all $i<n$.

EXAMPLE 3.3. The matroid $U_{n, 0}(q)$ is (by definition) $\mathrm{GL}_{n}(q)$-equivariantly realized by the collection of all hyperplanes in $\mathbb{F}_{q}^{n}$. The variety $\mathbb{P} X_{n, 0}(q)$ is an example of a Deligne-Lusztig variety for the group $\mathrm{GL}_{n}(q)$. The techniques developed by Lusztig [16] imply that the action of $\mathrm{GL}_{n}(q)$ on the cohomology group of $\mathbb{P} X_{n, 0}(q)$ is given by the unipotent $q$-analogue of Equation (3):

$$
\overline{O S}_{n, 0}^{i}(q) \cong V(q)\left[n-i, 1^{i}\right]
$$

for all $i<n$. See [6, Examples 6.1 and 6.4] for a concise and explicit statement of this result.

EXAMPLE 3.4. Let $M$ be any matroid, let $d \leqslant \operatorname{rk} M$ be a natural number, and let $M^{\prime}$ be the truncation of $M$ to rank $d$. Then $\overline{O S}_{M^{\prime}}^{*}$ is the truncation of $\overline{O S}_{M}^{*}$ to degree $d-1$. That is, we have a canonical isomorphism $\overline{O S}_{M^{\prime}}^{i} \cong \overline{O S}_{M}^{i}$ for all $i \leqslant d-1$, and $\overline{O S}_{M^{\prime}}^{i}=0$ for all $i \geqslant d$. In the case of Example 3.1, this reflects the fact that $\mathbb{P} X^{\prime}$ is a generic hyperplane section of $\mathbb{P} X$. In particular, we have

$$
\overline{O S}_{n, m}^{i} \cong V\left[n-i, 1^{i}\right] \quad \text { and } \quad \overline{O S}_{n, m}^{i}(q) \cong V(q)\left[n-i, 1^{i}\right]
$$

when $i<n-m$, and both groups are zero otherwise.

\section{KAZHDAN-LUSZTig POLYNOMials}

Let $M$ be a matroid on the ground set $E$ with lattice of flats $L$. For any $F \in L$, let $M_{F}$ denote the localization of $M$ at $F$; this is the matroid on the ground set $F$ whose bases are maximal independent sets of $F$. Let $M^{F}$ denote the contraction of $M$ at $F$. If $B$ is a basis for $M_{F}$, then $M^{F}$ is obtained from $M$ by contracting each element of $B$ and deleting each element of $F \backslash B$. Equivalently, $M^{F}$ is a matroid on the ground set $E \backslash F$, and $B^{\prime} \subset E \backslash F$ is a basis for $M^{F}$ if and only if $B^{\prime} \cup B$ is a basis for $M$. 
EXAMPLE 4.1. If $F$ is equal to the ground set of $M$ (the maximal flat), then $M_{F}=M$ and $M^{F}$ is the matroid of rank zero on the emptyset.

EXAMPLE 4.2. Proper (that is, non-maximal) flats of $U_{n, m}$ are subsets of $[n]$ of cardinality less than $n-m$. For such an $F,\left(U_{n, m}\right)_{F} \cong U_{|F|, 0}$ is Boolean, while $U_{n, m}^{F} \cong U_{n-|F|, m}$.

EXAMPLE 4.3. Proper flats of $U_{n, m}(q)$ are collections of linearly independent hyperplanes in $\mathbb{F}_{q}^{n}$ of cardinality less than $n-m$. For such an $F, U_{n, m}(q)_{F} \cong U_{|F|, 0}(q)$, while $U_{n, m}(q)^{F} \cong U_{n-|F|, m}(q)$.

The Kazhdan-Lusztig polynomial of $M$ is characterized by the following three conditions [7, Theorem 2.2]:

(1) If rk $M=0$, then $P_{M}(t)=1$.

(2) If rk $M>0$, then $\operatorname{deg} P_{M}(t)<\frac{1}{2} \operatorname{rk} M$.

(3) For every $M, t^{\text {rk } M} P_{M}\left(t^{-1}\right)=\sum_{F} \chi_{M_{F}}(t) P_{M^{F}}(t)$.

If $M$ admits the action of a finite group $W$, the equivariant Kazhdan-Lusztig polynomial is defined by the three analogous conditions, with the coefficients of the characteristic polynomial replaced by the graded pieces of the Orlik-Solomon algebra (with corresponding signs), which are now virtual representations of $W$ rather than integers. For every flat $F \in L$, let $W_{F} \subset W$ denote the stabilizer of $F$. If $C_{M, W}^{i}$ is the coefficient of $t^{i}$ in the $W$-equivariant Kazhdan-Lusztig polynomial of $M$ and $i<\operatorname{rk} M / 2$, we have the following explicit recursive formula [10, Proposition 2.9]:

$$
C_{M, W}^{i}=\sum_{\substack{[F] \in L / W \\ 0 \leqslant j \leqslant \mathrm{rk} F}}(-1)^{j} \operatorname{Ind}_{W_{F}}^{W}\left(O S_{M_{F}}^{j} \otimes C_{M^{F}, W_{F}}^{\mathrm{crk} F-i+j}\right),
$$

where we take in the sum one flat from each $W$-orbit in $L$.

EXAMPLE 4.4. Consider the case of the uniform matroid $U_{n, m}$. Proper flats are subsets of $[n]$ of cardinality less than $n-m$, and the $S_{n}$-orbit of a flat is determined by its cardinality. The stabilizer of a flat of cardinality $k$ is isomorphic to the Young subgroup $S_{k} \times S_{n-k} \subset S_{n}$. Thus Equation (6) transforms into the following recursion:

$$
\begin{aligned}
C_{n, m}^{i} & =(-1)^{i} O S_{n-m}^{i}+\sum_{k=0}^{n-m-1} \sum_{j=0}^{k}(-1)^{j} \operatorname{Ind}_{S_{k} \times S_{n-k}}^{S_{n}}\left(O S_{k, 0}^{j} \otimes C_{n-k, m}^{n-m-k-i+j}\right) \\
& =(-1)^{i} O S_{n-m}^{i}+\sum_{k=0}^{n-m-1} \sum_{j=0}^{k}(-1)^{j} O S_{k, 0}^{j} * C_{n-k, m}^{n-m-k-i+j}
\end{aligned}
$$

where the first term corresponds to the maximal flat $F=[n]$.

EXAMPLE 4.5. Consider the case of the $q$-uniform matroid $U_{n, m}(q)$. Proper flats are collections of linearly independent hyperplanes in $\mathbb{F}_{q}^{n}$ of cardinality less than $n-m$, and the $\mathrm{GL}_{n}(q)$-orbit of a flat is determined by its cardinality. The stabilizer of a flat of cardinality $k$ is isomorphic to the parabolic subgroup $P_{n, k}(q) \subset \mathrm{GL}_{n}(q)$. Thus Equation (6) transforms into the unipotent $q$-analogue of Equation (7):

$$
\begin{aligned}
C_{n, m}^{i}(q) & =(-1)^{i} O S_{n-m}^{i}(q)+\sum_{k=0}^{n-m-1} \sum_{j=0}^{k}(-1)^{j} \operatorname{Ind}_{P_{n, k}(q)}^{\mathrm{GL}_{n}(q)}\left(O S_{k, 0}^{j}(q) \otimes C_{n-k, m}^{n-m-k-i+j}(q)\right) \\
(8) \quad & =(-1)^{i} O S_{n-m}^{i}(q)+\sum_{k=0}^{n-m-1} \sum_{j=0}^{k}(-1)^{j} O S_{k, 0}^{j}(q) * C_{n-k, m}^{n-m-k-i+j}(q) .
\end{aligned}
$$


Proof of Theorem 1.2. By Equations (2), (3), and (4), Equation (8) is precisely the unipotent $q$-analogue of Equation (7). Then by Theorem 2.1, the first part of Theorem 1.2 is equivalent to the first part of Theorem 1.1. The second part of Theorem 1.2 follows from Theorem 2.1(2).

Acknowledgements. The author is indebted to June Huh for help with formulating the main result and to Olivier Dudas for help with proving it.

\section{REFERENCES}

[1] Scott Andrews, The unipotent modules of $\mathrm{GL}_{n}\left(\mathbb{F}_{q}\right)$ via tableaux, J. Algebraic Combin. 47 (2018), no. $1,1-15$.

[2] Clark T. Benson and Charles W. Curtis, On the degrees and rationality of certain characters of finite Chevalley groups, Trans. Amer. Math. Soc. 165 (1972), 251-273.

[3] Thomas Church, Jordan S. Ellenberg, and Benson Farb, FI-modules and stability for representations of symmetric groups, Duke Math. J. 164 (2015), no. 9, 1833-1910.

[4] Charles W. Curtis, Reduction theorems for characters of finite groups of Lie type, J. Math. Soc. Japan 27 (1975), no. 4, 666-688.

[5] Richard Dipper and Gordon James, On Specht modules for general linear groups, J. Algebra 275 (2004), no. 1, 106-142

[6] Olivier Dudas, Lectures on modular Deligne-Lusztig theory, in Local Representation Theory and Simple Groups, Series of Lectures in Mathematics, vol. 29, European Mathematical Society, 2018, pp. 107-177.

[7] Ben Elias, Nicholas Proudfoot, and Max Wakefield, The Kazhdan-Lusztig polynomial of a matroid, Adv. Math. 299 (2016), 36-70.

[8] Wee Liang Gan and John Watterlond, A representation stability theorem for VI-modules, Algebr. Represent. Theory 21 (2018), no. 1, 47-60.

[9] Alice L. L. Gao, Linyuan Lu, Matthew H. Y. Xie, Arthur L. B. Yang, and Philip B. Zhang, The Kazhdan-Lusztig polynomials of uniform matroids, https://arxiv.org/abs/1806.10852.

[10] Katie Gedeon, Nicholas Proudfoot, and Benjamin Young, The equivariant Kazhdan-Lusztig polynomial of a matroid, J. Combin. Theory Ser. A 150 (2017), 267-294.

[11] Katie R. Gedeon, Kazhdan-Lusztig polynomials of thagomizer matroids, Electron. J. Combin. 24 (2017), no. 3, P3.12 (10 pages).

[12] Thomas Hameister, Sujit Rao, and Connor Simpson, Chow rings of vector space matroids, https://arxiv.org/abs/1802.04241.

[13] Robert B. Howlett and Gustav I. Lehrer, Representations of generic algebras and finite groups of Lie type, Trans. Amer. Math. Soc. 280 (1983), no. 2, 753-779.

[14] Trevor K. Karn and Max D. Wakefield, Stirling numbers in braid matroid Kazhdan-Lusztig polynomials, https://arxiv.org/abs/1802.00849.

[15] Linyuan Lu, Matthew H. Y. Xie, and Arthur L. B. Yang, Kazhdan-Lusztig polynomials of fan matroids, wheel matroids and whirl matroids, https://arxiv.org/abs/1802.03711.

[16] George Lusztig, Coxeter orbits and eigenspaces of Frobenius, Invent. Math. 38 (1976), no. 2, 101-159.

[17] Peter Orlik and Louis Solomon, Combinatorics and topology of complements of hyperplanes, Invent. Math. 56 (1980), no. 2, 167-189.

[18] Nicholas Proudfoot and Ben Young, Configuration spaces, FS ${ }^{\circ}$-modules, and Kazhdan-Lusztig polynomials of braid matroids, New York J. Math. 23 (2017), 813-832.

Nicholas Proudfoot, University of Oregon, Department of Mathematics, Eugene, OR 97403, USA E-mail : njp@uoregon.edu 